City University of New York (CUNY) CUNY Academic Works

1987

\title{
Compactification of the Twisted Heterotic String
}

V. Parameswaran Nair

City College of New York

Alfred Shapere

University of California, Santa Barbara

Andrew Strominger

University of California, Santa Barbara; Princeton

Frank Wilczek

University of California, Santa Barbara

\section{How does access to this work benefit you? Let us know!}

More information about this work at: https://academicworks.cuny.edu/cc_pubs/377

Discover additional works at: https://academicworks.cuny.edu

This work is made publicly available by the City University of New York (CUNY).

Contact: AcademicWorks@cuny.edu 


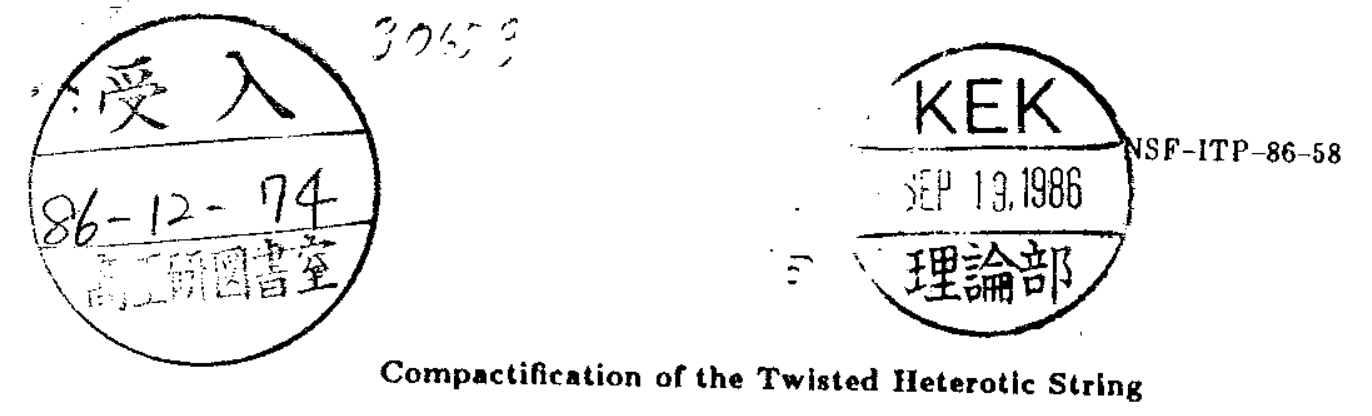

V.P. Nair !

Alfred Shapere ${ }^{2}$

Andrew Strominger ${ }^{1,3}$

Frank Wilczek ${ }^{1,2}$

${ }^{1}$ Institute for Theoretical Physics

University of Californis
Santa Barbarn, Californis 93106

${ }^{2}$ Department of Physics

Univesity of Califomis

Santa Barbara, Califomia 93106

${ }^{3}$ Institute for Adranced Study

Princeton, NJ 08540

\section{ABSTRACT}

We consider compactifications of the recently discovered tachyou free non. supersym-me'ric string on tori of Lorentzian self dual lattices. The one loop cosmological constant is computed as a function of the parameters characterixiag the lattices. When the lattice parameters are treated as dynamical variables, their equations of motion require extremization of $\Lambda$. This extremization determines the allowed gauge groups and mass spectra. We explicitly find some extrems of $\Lambda_{1}$ ralid to all orders in perturbation theory. $A$ very general duality relation between compactifications is demonstrated, which leads to equiralence of compactifications on tori of radii $R$ and $k$.

\section{Introduction}

Recent progress in understanding string theories bas brought to light a number of new constructions for producing both uew string theories $[1-3]$ and new compacti. fications $|1-6|$. Whether these constructions truly differ in a fundamental way, or are merely expansions of a single (or a few) string field theories around different racua is an important issue, for hope of deriving observable realities from considerations of consistency at the Planck mass must largely rely on there being a sererely limited choice of options. To settle this issue it will ultimately be necessary to go well beyond perturbative expansions, and to determine if constructions which look quite different in perturbation theory are somehow connected. Even if they do turn out to be con. nected to one another an important practical question will remain: which racuum, if any, is the best starting point for a perturbative expansion? This is at lest partially a question of stability; the false vacila will decay to the correct one.

In this note we combine two of the new constructions. to define still more consis. tent compactifications. The nature of these compactifications permits us to make a modest start ou the questions of connectedness and stability. Specifically, we combine Narain's idea of compactification on Lorentzian lattices $[4]$ with the recent ideas of Dixon and Harvey (1], and of Alvarez-Gaumé, Ginsparg. Moore, and Vafa $|2|$ for constructing non-supersymmetric string theories. Narain's construction leacts to classes of models which are continuously connected to one another. Indeed, this construction has been reinterpreted $[7]$ as the presence of certain constant background fields on the 16-dimensional tons of the usual beterotic string [8]. It is very reasonable, then. to think that these modelo all sit within the same class - namely they are all different compactifications of the heterotic string. At the same time, the twisting construction has the importaut new feature of producing tachyon free models without supersym. metry. In particular, a finite non-zero cosmological term appears in these models due to imperfect cancellation between boson and fermion zero point energies.

When we combine the two constnuctions, we find that the magnitucle of the cos. 
mological term depends on the form of the Loreutzian lattice for, equivalently, on the magnitudes of the background fields). Stability requires that this term be extremized. This oceurs only for a discrete set of compastifications.

A crucial feature of our analysis is a remarkable duality between tori with radii $\boldsymbol{R}$ and $\frac{\hbar}{k}$ (in units of string tension). We show that this duality implies a complete isomorphism between $S$ - matrix elements on the different tori and bas a generalization to arbitrary Lorentzian even self dual lattices. This duality is intimately related to several other dualities: the classic $\leftrightarrow t$ channel duality and modular invariance, Kramers-Wannier duality of two-dimensional theories [9|, and electric-magnetic or charge-monopole duality [10]. In the present context, this duality is especially important because it allows us to identify stationary lattices, i.e. lattices for which the cosmological term has zero derivative to all onders in string perturbation theory.

\section{The twisted heterotic atring on Lorentrian lattices}

We begin with a brief review of Narain's heterotic string theories and the twisted ten dimensional heterotic string. Then, after defining a twist projection and enumerating the sectors in twisted lower-dimensional theories, we compute the partition function and the cosmological constant $\Lambda$. Asymptotic properties of $\Lambda$ are discussed, and local extrema are found. Modular invariance is proved in the appendix.

In the ten dimensional heterotic string [8], a 26-dimensional left moving closed bosonic string is fused to a right moving 10-dimensional closed superstring. The sixteen "interna" left moving dimensions are compactified on a 16 -torus $R^{10} / \Gamma$, where the lattice $\Gamma$ is required to be integral, even and self dual by momentum quantization, o-translation invariance, and modular invariance, respectively. Such lattices exist only in 8 n dimensions, and there are only two in 16 dimensions. These are the root lattices of $E_{3} \times E_{3}$ and $S \operatorname{pin}(32) / Z_{2}$. Actions of these groups on the atring Hibert space are realized when the internal dimensions are compactified on the corresponding tori. Remartably, these same groups are singled out by the requirement that the resulting low energy feld theory be anomaly free [11].
Narain realized that the heterotic construction may be extended to dimensions $D<10$ by compactifying $(26-D)$ left moving and $(10-D)$ right moving string degrees of freedom on a Lorzatzian even self dual lattice $\Gamma$ in $R^{26-D, 10-D}$ (4). Clasaically, the compactified left and right movers then bave the mode expansions

$$
\begin{aligned}
& x^{I}(\sigma+t)=x^{I}+L^{I}(\sigma+t)+\sum_{n \neq 0} \frac{i}{2 n} a_{n}^{l} e^{i n(\sigma+t)} \\
& \dot{x}^{A}(\sigma-t)=\bar{x}^{A}+\dot{L}^{A}(\sigma-t)+\sum_{n \neq 0} \frac{i}{2 n} a_{n}^{A} e^{i n(\sigma-t)}
\end{aligned}
$$

where $\left(L^{I}, \dot{L}^{\Lambda}\right) \in \Gamma$ is the winding vector, and $a$ ranges from 0 to $2 \pi$. In the quantum theory, the left and right moving mass operators are

$$
\begin{aligned}
& \frac{1}{4} m_{L}^{2}=\frac{1}{2} L^{2}+N-1 \\
& \frac{1}{4} m_{R}^{2}=\frac{1}{2} \dot{L}^{2}+\dot{N}-c
\end{aligned}
$$

$c$ is a normal ordering constant which equals 0 for the Ramond sector and $\frac{1}{2}$ for the Neveu-Schwarz sector. The physical states obey the constraint

$$
m_{L}^{2}=m_{R}^{2}
$$

Modular invariance requires that the tachyon at level $-\frac{1}{2}$ be projected out of the physical spectrum. Thus $m_{R}^{2}$ is never negative and since $m^{2}=m_{L}^{2}+m_{R}^{2}=2 m_{R}^{2}$. these theories are tachyon free.

The massless gauge boson states correspond to winding vectors $(L, 0) \in \Gamma$ with $L^{2}=2$ in addition to the standard Kaluza-Klein $U(1)$ gauge bosons. If the set of such winding vectors contains the root lattice of a Lie group $G$, then $G$ is a gauge group of the theory. The Kaluza-Klein $U(1)$ bosons correspond to the Cartan subalgebra of the gauge group. Narain has found lattices leading to several gauge groups, including $S O(52-2 D)$ and $E_{8} \times E_{8} \times G_{10-D}$ where $G_{10-D}$ is any simply laced Lie group of rank $(10-D)$

The above coustruction gives a $(26-D)(10-D)$ parameter family of compactifications of the heterotic atring to $D$ dimensions. This is because any two Loreutzian 
even self dual lattices in $\mathbf{R}^{26-D, 10-D}$ are related by an $S O(26-D, 10-D)$ rota. tion. In general, such rotations will change the mase spectrum, unless both $m_{L}^{2}$ and $m_{R}^{2}$ are left unchanged. Physically distinct sompactifications therefore correspond to "Lorents boosts," i.e., points in the manifold

$$
M=\frac{S O(26-D, 10-D)}{S O(26-D) \times S O(10-D)}
$$

The other modification of the beterotic string mentioned above is obtained by defining a "twisted fermion number operator" $\alpha$, with $\alpha^{2}=1$, and projecting in a modular inrariant fashion onto states with $\alpha=1$. The $a$ defined in $[1]$ is

$$
a=(-1)^{G} \cdot e^{2 x i-6}
$$

where $2 \delta \in \mathrm{r}$ and $\mathrm{G}$, the G-parity, is defined as follows. In the antiperiodic (NeveuSchwarz) nector constructed from oucillators of half-integral level number, $G$ is defined to be worldsheet fermion number minus one. In the periodic (Ramond) sector, $G$ is the chirality operator \%. The GSO projectian corresponds to $\alpha=(-1)^{G}$ and yields the original heterotic string. Adding in the extra $\mathrm{Z}_{2}$ translation in the compactification tonus leads to radically different theories.

The particular choice of $\delta^{2}=2$, realized by

$$
\delta_{0}=\left\{\begin{array}{lll}
\left(1,0^{7} ; 1,0^{7}\right) & \text { in } \Gamma_{8} \oplus \Gamma_{8} \\
\left(\left(\frac{1}{2}\right)^{8}, 0^{8}\right) & \text { in } \Gamma_{16}
\end{array}\right.
$$

results in a chiral, tachyon free theory with $O(16) \times O(16)$ symmetry and no spacetime supersymmetry. Briefly, the projection $\frac{1}{2}(1+a)$ applied to either of the original heterotic strings results in two sectors: an even G-parity Neveu-Scliwarz bosonic sector with $\delta \cdot L \in \mathbf{Z}$ and a Ramond sector with $\delta \cdot L \in \mathbf{Z}+\frac{1}{2}$ (and negative helicity, with our convention for $G$ ). In addition, modular invariance demands that we include two twisted sectors of string which close only up to a transformation by $\alpha: X(2 \pi)=$ $a X(0) a^{-1}$ (which amounts to replacing $L$ by $L+\delta$ in the mode expansion). These are an NS sector with $\delta \cdot L \in \mathrm{Z}+\frac{1}{2}$, and a $\mathrm{R}$ sector with $\delta \cdot L \in \mathrm{Z}$. The only remaining symmetry group generators correspond to $L^{2}=2$ with $\delta \in Z$ and $G=O(16) \times O(16)$. Furthermore, the lowest mass level in the left moving twisted sector is at $f m_{L}^{2}=\frac{1}{2}(L+\delta)^{2}+N-1=\frac{1}{2} \delta^{2}-1$, so $\delta^{2}=2$ and the constraint (2) ensure that the theory contains uo tachyon.

Now consider applying such a twist to the theories of Narain. If $\Gamma$ is a Lorentzian lattice, let

$$
a \equiv(-1)^{G}(-1)^{2(L \cdot \delta-i \cdot j)}
$$

where $(2 \delta, 2 \dot{\delta}) \in \Gamma$ and $\delta^{2}-\dot{\delta}^{2} \in \mathbf{Z}$. In particular, we shall focus on the case $\delta^{2}-\tilde{\delta}^{2}=2$. which alway leads to a tachyon free theory. Such vectors $(\delta, \delta)$ always exist, becaluse any Lorentzian lattice is an $S O(26-D, 10-D)$ rotation of $\Gamma_{8} \oplus \Gamma_{8} \oplus(10-D) \cdot \sigma_{1}$. (Here $\sigma_{1}$ is the Lorentzian lattice in $R^{1,1}$, generated by the null vectors $\frac{1}{\sigma}(\dot{t} \pm \dot{i})$.) We can just take $(\delta, \delta)$ to be the very same rotation of the $\delta_{0}$. Other such twists for $\Gamma_{\mathrm{g}} \oplus \Gamma_{\mathrm{g}} \oplus(10-D) \sigma_{1}$ are possible, such as

$$
\begin{aligned}
& \delta_{1}=\left(\sqrt{\left.1,0^{7} ; 0^{8} ;\left(\frac{1}{\sqrt{2}}\right)^{2}, 0^{8-D}, 0^{10-D}\right)}\right. \\
& \delta_{2}=\underbrace{(\underbrace{10}}_{0^{8} ; 0^{8} ;\left(\frac{1}{\sqrt{2}}\right)^{4} \cdot 0^{6-D} \cdot 0^{10-D}})
\end{aligned}
$$

and $\delta_{0}, \delta_{1}$ or $\delta_{2}$ plus any perpendicular light-like vector twice which is in $\Gamma$. These exhaust the inequivalent twists with $\delta^{2}-\dot{\delta}^{2}=2$. In addition, there are other inequir. alent twists with $\delta^{2}-\dot{\delta}^{2} \neq 2$ leading to tachyon free theories. $\left(\delta^{2}-\dot{\delta}^{2}\right.$ is in general constrained to be an integer in order to have matched left and right moving maso levels in accordance with equation (2).) However, in what follows, we consider only $\delta^{2}-\dot{\delta}^{2}=2$

Like the $O(16) \times O(16)$ string, these theories all have iwisted and untwisted fermionic and bosonic sectors. We shall enumerate the states with $a=1$ in each of these four sectors. The untwisted bosonic sector is the product of a rigbt moving even G-parity NS rector and a left moving bosonic sector witb $L \cdot \delta-\dot{L} \cdot \dot{\delta} \in \mathbf{Z}$. So 
the untwisted bosonic partition function is

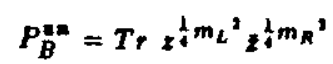

$$
\begin{aligned}
& =\frac{1}{22^{\frac{1}{2}} x} \prod_{n=1}^{\infty}\left(\frac{1}{1-z^{n}}\right)^{24}\left[\prod_{n=1}^{\infty}\left(\frac{5+z^{n+\frac{1}{2}}}{1-z^{n}}\right)^{8}-\prod_{n=1}^{\infty}\left(\frac{1-z^{n-\frac{1}{2}}}{1-z^{n}}\right)^{8}\right] \\
& \sum_{(L, \dot{L}) \in \mathrm{r}} z^{\frac{1}{L^{\prime}} z^{\frac{1}{2}} \dot{L}^{\prime}} \frac{1}{2}\left(1+(-1)^{2(L \cdot \delta-i \cdot \delta)}\right)
\end{aligned}
$$

where the trace is over mass eigenstates in the projected sector. In this expression,

$$
\frac{1}{22^{\frac{1}{2}} z} \prod_{n}\left(\frac{1}{1-2^{n}}\right)^{24}\left[\prod_{n}\left(\frac{1+z^{n-1}}{1-z^{n}}\right)^{8}-\prod_{n}\left(\frac{1-z^{n-\frac{1}{2}}}{1-z^{n}}\right)^{8}\right]
$$

is the usual partition function of the bosonic (NS) sector of an uncompactified heterotic string, with a minus sign in the bracketed factor for projection onto even G-parity atales. The compactification is accounted for by the sum over lattice vectors, with the projection operator

$$
\frac{1}{2}\left(1+(-1)^{2(L-\delta-\dot{L} \cdot \dot{\delta})}\right)
$$

erring to pick out states with $L \cdot \delta-\dot{L}, \dot{\delta} \in \mathbf{Z}$.

The untwisted fermions have $L \cdot \delta-\dot{L} \cdot \tilde{\delta} \in \mathbf{Z}+\frac{1}{2}$ so similarly

$$
P_{F}^{* n}=\frac{8}{2} \prod_{n=1}^{\infty}\left(\frac{1}{1-z^{n}}\right)^{24}\left(\frac{1+z^{n}}{1-E^{n}}\right)^{8} \sum_{\Gamma} z^{\frac{1}{3} L^{2} z^{\frac{1}{2} L^{\prime}}} \frac{1}{2}\left(1-(-1)^{2(L \cdot \delta-\dot{L} \cdot \dot{\delta})}\right)
$$

In the twisted NS sector, modular invariance will require projection onto states with balf-integer level number, i.e., odd G-parity. Modular invariance will also imply that the path integral over the twisted NS sector must be summed with weight +1 , indicatiog that this sector is bosonic. The mass operators are also different, since compactified momenta now lie in the shifted lattice $\Gamma+\delta$ :

Therefore

$$
\begin{aligned}
& \frac{1}{4} m_{L}^{2}=\frac{1}{2}(L+\delta)^{2}+N-1 \\
& \frac{1}{4} m_{R}^{2}=\frac{1}{2}(\dot{L}+\delta)^{2}+\dot{N}-\frac{1}{2}
\end{aligned}
$$

$$
\begin{aligned}
P_{B}^{t w} & =\frac{1}{2 z^{\frac{1}{2} z}} \prod_{n=1}^{\infty}\left(\frac{1}{1-z^{*}}\right)^{24}\left[\prod_{n=1}^{\infty}\left(\frac{1+z^{n-\frac{1}{2}}}{1-z^{n}}\right)^{8}+\prod_{n=1}^{\infty}\left(\frac{1-z^{n-\frac{1}{2}}}{1-z^{n}}\right)^{8}\right] \\
& \sum_{(L, i) \in \Gamma} z^{\frac{1}{2}(L+\delta)^{2} \sum_{z} \frac{1}{2}(\dot{L}+\delta)^{2}} \cdot \frac{1}{2}\left(1-(-1)^{2(L \cdot \delta-\dot{L} \cdot \delta)}\right)
\end{aligned}
$$

Similarly,

$$
\begin{aligned}
p_{F}^{t w} & =\frac{8}{z} \prod_{n=1}^{\infty}\left(\frac{1}{1-z^{n}}\right)^{2 \alpha}\left(\frac{1+z^{n}}{1-z^{n}}\right)^{8} \sum_{(L, \dot{L}) \in \Gamma} z^{\frac{1}{2}(L+\delta)^{2}} z^{\frac{1}{1}(i+j)^{2}} \\
& \cdot \frac{1}{2}\left(1+(-1)^{2(L \cdot \delta-L \cdot \delta)}\right)
\end{aligned}
$$

Putting everything together and using the atandard 8-function identity $14+14=14$ we find

$$
\begin{aligned}
& P_{B}-P_{F}=\frac{1}{z} \prod_{n=1}^{\infty}\left(\frac{1}{1-z^{n}}\right)^{24}\left\{8 \prod_{n=1}^{\infty}\left(\frac{1+z^{n}}{1-z^{n}}\right)^{d} \sum_{\Gamma} z^{1} L^{1} z_{2}^{1} L^{3}(-1)^{2(L \cdot d-L \cdot b)}\right.
\end{aligned}
$$

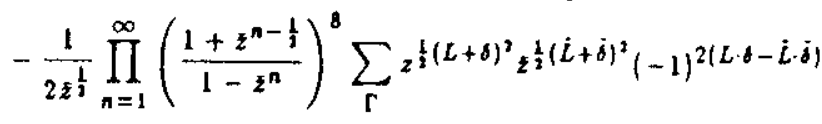

$$
\begin{aligned}
& \left.+\frac{1}{2 z^{\frac{1}{3}}} \prod_{n=1}^{\infty}\left(\frac{1-z^{n-\frac{1}{2}}}{1-z^{n}}\right)^{8} \sum_{\Gamma} z^{\frac{1}{2}(L+\delta)^{3} z^{\frac{1}{2}}(\dot{L}+\delta)^{3}}\right\}
\end{aligned}
$$

We now discuss the massless sectors of these theories. First consider the untwisted bosonic sector, with $|0\rangle=|0\rangle_{L}|0\rangle_{R}$ the product of the left moring vacuum with $N=1$ and the right moving Neveu-Schwarz racuum at level $\dot{N}=-\frac{1}{2}$. The lowest lying states satisfying the constraint $m_{L}^{2}=m_{R}^{2}$ are the massless states

$$
\begin{array}{cc}
a_{-1, L^{b}}^{i} b_{-\frac{1}{2}, R}^{j}|0\rangle & a_{-1, L^{b}}^{i}{ }_{-\frac{1}{2}, R}|0\rangle \\
a_{-1, L^{b}}^{A} b_{-\frac{1}{2}, R}^{j}|0\rangle & a_{-1, L^{b}}^{A} b_{-\frac{1}{2}, R}^{I 0\rangle} \\
b_{-\frac{1}{2}, R}^{j}|(L, 0)\rangle & \left.b_{-\frac{1}{2}, R}^{l} \mid(L, 0)\right) \\
(2 \leq i<D, D \leq I<10, D \leq A<26)
\end{array}
$$

where $|(L, 0)\rangle$ is the lowest state with $\delta \cdot L \in \mathrm{Z}, L^{2}=2$, and $(L, 0) \in \Gamma$. The $\alpha^{\prime}$ are left moving bosonic oscillators and $b$ 's are right moring fermionic oscillators. The untwisted fermionic states, with the Ramond racuum transforming as an 8 , under $O(8)$, are simply

$$
|\mathbf{a},(L, 0)\rangle
$$

where now $\delta \cdot L \in Z+\frac{1}{2}, L^{2}=2$ and $a$ is the 8 , index.

The twisted NS sector contains the massless scalar bosous

$$
((L+\delta, \dot{L}+\dot{\delta}))^{\prime}
$$


where $(L+\delta)^{2}=2 \cdot(\dot{L}+\dot{\delta})^{2}=1$ and $L \cdot \delta-\dot{L} \cdot \dot{\delta} \in \mathrm{Z}+\frac{1}{2}$. (We label all twisted states with a prime.) The twisted $R$ aector fermions transform as an $8_{\mathbf{a}^{\prime}}$ :

$$
\left|a^{\prime},(L+\bar{\delta}, 0)\right\rangle^{\prime}
$$

where $(L+\delta)^{2}=2, L \cdot \delta \in \mathbf{Z}$, and $(L, 0) \in \Gamma$. Note that the massless state spectrum is nonchiral for $D<10$.

As an example, consider the Inttice $\Gamma_{\max }$ generated by the rectors in $\mathrm{R}^{26-D, 10-D}$

$$
\begin{gathered}
\left( \pm e_{i} \pm e_{j}, 0\right) \quad 1 \leq i<j \leq 26-D \\
\left(0, \pm \dot{e}_{j} \pm \bar{e}_{j}\right) \quad 1 \leq i<j \leq 10-D \\
\left(w_{0}, \dot{w}_{v}\right)=\left(\frac{1}{2}\left(e_{1}+\ldots+e_{2 b-D}\right), \frac{1}{2}\left(\dot{e}_{1}+\ldots+\dot{e}_{10-D}\right)\right) \\
\left(w_{v}, \dot{\omega}_{v}\right)=\left(e_{1}, \dot{e}_{1}\right)
\end{gathered}
$$

The rectors $(k, 0)$ in $\Gamma_{\max }$ with $k^{2}=2$ generate the root lattice of $S O(52-2 D)$, which is therefore the gauge group of the heterotic string theory compactified on $\Gamma_{\max }$. $\left(\omega_{0}, \dot{\omega}_{0}\right)$ and $\left(\omega_{v}, \dot{w}_{v}\right)$ are spinor and vector weights of $S O(52-2 D), S O(20-2 D)$. For the twist $(\delta, \delta)=\left(\left(\frac{1}{2}\right)^{8} ; 0^{s} ; 0^{10-D} ; \tilde{0}^{10-D}\right)$ this group is broken down to $G=$ $O(16) \times O(36-2 D)$. The massless field theory bas bosons which transform under $O(D-2) \times O(16) \times D(36-2 D)$ ss

$$
\mid(10-D) \cdot(1,1,1) \oplus(D-2,1,1)]_{R} \otimes\left[\left.(D-2,1,1) \oplus(1, A d j, 1) \oplus(1,1, \text { Adj })\right|_{L}\right.
$$

These bosons include $O(16) \times O(36-2 D)$ gauge fields as well as $(10-D)$ scalars in the edjoint of $G,(10-D) U(1)$ vector bosons, and $g^{\mu \nu}, B^{\mu \nu}$ and $\phi$. The twisted and untwisted fermions transform under $O(8) \times O(16) \times O(36-2 D) *$ as

$$
(8,16,36-2 D) \quad \text { and } \quad(8,128,1)
$$

for $D<10$. What about other twists? If $(\delta, \dot{\delta})=\left(\left(\frac{1}{2}\right)^{9}, 0^{18-D} ; \frac{1}{2}, 0^{9-D}\right)$, then the maximal symmetry group is $O(18) \times O(34-2 D)$, and the massless twisted sector disappears. Clearly, by choosing appropriate twists, we can get any maximal aymmetry - Of course, $O(8)$ is broken down to $O(D-2)$ but it is more convenient notationally to group the $O(D-2)$ representations together. group

$O(2 n) \times O(2 m)$, where $2 m, 2 n \geq 16$ and $n+m=26-D$

The methods of reference 12 allow us to write down the cosmological constant for the compactified theory without furtber ado. Let $F$ be the fundamental region $\left\{r=r_{1}+i r_{2} \in \mathbf{C}: r_{2}>0,\left|r_{1}\right|<\frac{1}{2},|r| \geq 1\right\}$. Then the cosmological constant in a ten dimensional uncompactified theory is

$$
\Lambda_{1-\text { loop }}=-c \int_{F} \frac{d^{2} r}{r_{2}} r_{2}^{-5}\left|P_{B}(z, z)-P_{F}(z, z)\right|
$$

where $c$ is a positive numerical factor which depends on the atring tension and the factor $r_{2}^{-5}$ arises from the integration over $D$-dimensional momenta for $D=10$. In our case, we simply replace $r_{2}^{-3}$ with $r_{2}^{-D / 2}$ and obtain

$$
\Lambda_{1-\text { loop }}=-c^{\prime} \int_{F} \frac{d^{2} r}{r_{2}^{2}} \quad r_{2}^{-(D-2) / 2}\left\{P_{B}(z, \bar{z})-P_{F}(z, \bar{z})\right\}
$$

We now turn to the question of modular invariance of $A$. Our argument will be along the lines of reference 3 and details are in the appendix. The partition function (8) can be written as

$$
P=-B \sum_{\alpha \beta} \lambda_{\alpha \beta} C_{\alpha \beta}
$$

where

$$
B=z^{-1} z^{-1} \prod_{n} \frac{1}{\left(1-z^{n}\right)^{24}\left(1-z^{n}\right)^{8}}=\eta(r)^{-24} \overline{\eta(r)}^{-8}
$$

and $\eta$ is the Dedekind $\eta$ function. The $A_{a \beta}$ 's are in the notation of reference 15 , i.e., $(\alpha \beta)$ specifies $(\sigma, t)$ bcindary conditions with (anti-) periodicity denoted by $+(-)$ :

$$
\begin{aligned}
& A_{--}=\frac{1}{2} z^{-\frac{1}{6}} \prod_{n}\left(1+z^{n-\frac{1}{1}}\right)^{8} \\
& A_{+-}=-8 z^{\frac{1}{8}} \prod_{n}\left(1+z^{n}\right)^{8} \\
& A_{-+}=-\frac{1}{2} z^{-\frac{1}{6}} \prod_{n}\left(1-z^{n-\frac{1}{2}}\right)^{8}
\end{aligned}
$$

The bar over $A_{\alpha A}$ denotes complex conjugation. The $C_{n^{\prime}} y^{\prime}$ are sums over lattice 
rectors with twists put in.

$$
\begin{aligned}
& C_{--}=\sum z^{\frac{1}{2}(L+\delta)^{2}} z^{\frac{1}{2}(\dot{L}+\dot{\delta})^{2}}(-1)^{2(L \cdot \delta-\dot{L} \cdot \dot{\delta})}
\end{aligned}
$$

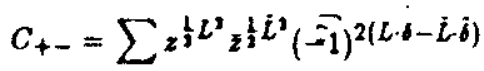

$$
\begin{aligned}
& C_{-+}=\sum_{8} \frac{1}{2}(L+6)^{2}{ }^{1} \frac{1}{2}(\dot{L}+\delta)^{\prime}
\end{aligned}
$$

The correspondence for the $C$ 's is as follows: in the $(\sigma, t)$ notation, antiperiodicity in $\sigma$ corresponds to summing over lattice vectors with a shift, i.e. $(L, i) \rightarrow(L+\delta, \dot{L}+$ $\dot{\delta})$, while antiperiodicity in $t$ corresponds to the insertion of the factor $(-1)^{2(L \cdot \delta-\dot{L} \cdot \dot{\delta})}$.

From the amplitudes $\lambda_{a \beta}$ and $C_{\alpha} \beta^{\prime}$ one can construct 16 possible products. Certain eppropriately weighted sums of these products correspond to unitary, modular invariant string theories $[1-3]$. In our case, the partition function is the sum of the diaganal terms, where the spin structure $(\alpha \beta)$ is identified with the twist structure $\left(\alpha^{\prime} \beta^{\prime}\right)$ of the lattice sums. From the transformation of the amplitudes given in the appendix, $\boldsymbol{\Lambda}$ is easily verified to be modular invariant.

There are also other combinations of the 16 amplitudes which are modular inrariant, e.g., $\left(\sum A_{\alpha \beta}\right)^{\prime} \cdot\left(\sum C_{\alpha^{\prime} \beta^{\prime}}\right)$. This is zero since $\sum A=0$. Since the diagonal oum is modular invariant, we see that the nondiagonal terms in the above sum also give a modular invariant combination corresponding to a theory with a cosmological constant of the opposite sign to the diagonal one. This theory has tachyons, however, and we shall not discuss it further.

We shall conclude this section with some remarks on the question of modular invariabce for higher loop amplitudes. For $\ell$ loop string calculations, we have to consider sums over surface with $2 \ell$ noncontractible loops or homology cycles. This gives $2^{2 \ell}$ spin structurei or choices for fermionic boundary conditions. The difference between two spin structures corresponds to the assignment of reiative \pm signs corresponding to periodicity or antiperiodicity. Since the values of $X$ differing by lattice vectors are identifed, one can also associate winding numbers (lattice vectors) and twist vectors with each cycle. There is thus a one to one correspondence between spin structures and twist struetures; for each spin strucure, there is a corresponding twist structure for the lattice sums and the amplitudes $A_{a_{1}, \beta_{1} \ldots}$ and $C_{\sigma_{1}^{\prime}, \delta_{1}^{\prime}} \ldots$ can be constructed. Presumably the diagonal sum will give a modular invariant amplitude.

\section{Extrema of $\wedge$ and equations of motion}

In section II we bave described $(26-D)(10-D)$ parameter families of string compactifications hich are degenerate at the string tree level. We nom wish to determine which of these temain solutions at the nae lonp level. In terms of atring diagrams, this requires that the one loop one-point functions ranish [13]. These are given by the expectation values of vertex operators on the world sheet torus. In principle, one might have to consider an infinite number of rertex operaton. In practice this is avoided becsuse we have s small parameter st our disposal, namely the dilaton expectation value $\phi_{0}$, which governs the strength of string loop corrections. At string tree level, this is a free parameter, and it must be assumed to be small for the consistency of our analysis. This small paraneter justifies ignoring one point functions for massive fields - they at most shift their expectation values by a smal! amount." The relevant massless modes are the lattice moduli (which correspond to deformations of the metric and the gauge and antisymmetric fields (7]) and the ten dimensional dilaton which couples to the Euler density of the world sheet manifold.

Consider first the $(26-D)(10-D)$ vertex operators that create the masoles modes associated with the lattice moduli. In general, the one point function associated with a massless deformation of the background is simply the linearized rariation of the functional integral under the deformation. In our case, the one point functions ranish at $\Gamma=\Gamma_{0}$ if

$$
\left.\frac{\delta}{\delta \Gamma} \Lambda(\Gamma)\right|_{\Gamma=\Gamma_{0}}=0
$$

We shall sbow that $\Lambda$ has several critical points as a function on the lattice moduli space $S O(p, q) / S O(p) \times S O(q)(p=26-D, q=10-D)$, a consequence of a certain duality relating the different theories on this space. - In particular one can ignore at this order the tadpole which might sell us to shift to
de Sitter space $|14|$. The scale of the relevant de Sitter geometry will be large relative
to the string scale. 
As usua, let $\Gamma$ be a Lorentzian lattice with $(L, \dot{L}) \in \Gamma$. We consider ane parameter family of $S O(p, q)$ transformations which mix the vectors $L$ and $\dot{L}$. We shall first examine the case of compactificatoon to $D=9$, i.e. $q=1$. In this case $L_{i}$ is 17 dimensional rector and we can write

$$
\begin{aligned}
& L_{i}^{\prime}=\cosh \beta_{i} L_{i}+\sinh \beta_{i} \dot{L} \\
& \dot{L}^{\prime}=\sinh \beta_{i} L_{i}+\cosh \beta_{i} \dot{L}
\end{aligned}
$$

$\beta_{i}$ parameterizes the "boost" which mixes the $i$ th component of $L$ with $\dot{L}$.

$\Gamma$ is an extremal point of $\Lambda$ if $\Lambda\left(\Gamma\left(\beta_{i}\right)\right)=\Lambda\left(\Gamma\left(-\beta_{i}\right)\right)$ for infinitesimal $\beta_{i}$ for all 17 choices of $i$, since these 17 boosts form a local basis for the space $\frac{S O(17,1)}{S O(17) \times S O(1)}$. From the transformation equations above, we see that $\beta_{i} \rightarrow-\beta_{i}$ can be compensated for by $\dot{L} \rightarrow-\dot{L}$. Since the expression for the masses is invariant under $\dot{L} \rightarrow-\dot{L}, \Gamma$ will be a critical point of $\Lambda_{1-100}$ provided $(L,-\dot{L})$ is always a lattice vector. (Then the summation $\sum_{L, i}$ also runs over the same range.)

Consider now the general case of compactification to $D$ dimensions. The lattices may now be parametrized by $S O(p, q)$ elements. We shall call lattices symmetric if $(L, \dot{L}) \in \Gamma \Leftrightarrow(L,-\bar{L}) \in \Gamma$, and denote them by $\Gamma_{\text {sym }}$. (Note that a Lorentzian self dual lattice is symmetric if and only if it is also Euclidean seif dual (although it need not be Euclidean even). Hence the number of symmetric lattices in D dimensions is finite.) For $S \in S O(p, q)$, let $\Gamma_{\text {rym }}(S)$ be the lattice obtained by boosting $\Gamma_{\text {sym }}$ by $S$. It is atraightforward to see that $\Lambda_{1-100 p}\left[r_{\text {xym }}\left(S^{T}\right)\right]=\Lambda_{1-\text { loop }}\left[\Gamma_{\text {sym }}\left(S^{-1}\right)\right]$. Also, from the definition of $\Gamma_{\mathrm{gym}}$ that for $S$ near the identity, the vector in $\frac{S O(p, Q)}{S O(p) \times S O(q)}$ corresponding to $S^{-1}$ is minus that corresponding to $S^{T}$. Thus $\Gamma_{\text {sym }}$ is an extremum of $A_{1-1009}$

For the lattice $\Gamma \max$ of equation (9) leading to the maximal gauge group, we have weights of $S O(4 k)$ for even $D$, where $2 k=26-D$. In this case, if $\left(w_{0}, w_{0}\right)$ is in $\Gamma$, then so is $\left(w_{0},-\bar{w}_{0}\right) . \Gamma_{\max }$ is thus symmetric and is a critical point of $\Lambda_{1-\text { ioop }}$. Another critical point is the lattice $\Gamma_{8} \oplus \Gamma_{8} \oplus n \sigma_{1}$, which corresponds to the gauge sroup $O(16) \times O(16) \times S U(2)^{n}$. In the next section, we shall show that the theories defined by $\Gamma_{5 y m}\left(S^{T}\right)$ and $\Gamma_{5 y m}\left(S^{-1}\right)$ formally bave the same cosmological constant to all orders in string perturbation theory and so symmetric lattices are extrem to all orders as well.

The formulation of these theories in terms of constant background values of mase. less fields also leads us to expect the existence of a large number of critical points for $\mathrm{A}$. The topology of the moduli space is quite complicated aince there are various periodic shifts of the background fielda which leave the theory physically unchanged This means that the Betti numbers of the moduli space will be large and Morse theory suggests a correspondingly large number of critical points.

We now turn to the ten dimensional dilaton. Its one loop one point function vanishes if and only if the cosmological constant $\Lambda$ does. $\Lambda=0$ at the string tree level by virtue of the conformal invariance of the $\sigma$-model [13]. Since the one loop cosmological constant is generically nonzero, our approximate solutions will in general develop some slow (for small $\phi$ ) time dependence controlled by the value of $A$ at the extrems. For $\Lambda$ positive, will roll in to weak coupling, while if $\Lambda$ is negative it will roll to strong coupling. Nevertheless for small $\phi$ we have approximate solutions for a long time.

Of particular interest is the point at "infinity" corresponding to "decompactifica. tion." By this we mean the limit in which all the radii of the $(10-D)$ diruensional compactification torus go to infinity, while other parameters are held finite. (This limit is most easily understood if one interprets the Lorentrian lattice compactifications in terms of expectation values for background massless felds. It then corresponds to an infinite constant rescaling of the metric.) We will show that this point is a local minimum of the $D$ dimensional energy. For large radii, the field theory approximation should be ralid. The dynamics are described by the ten dimensional effective action

$$
S_{10}=\int d^{10} x \sqrt{-g^{(10)}}\left(R^{(10)}-\Lambda^{(10)}\right)
$$

where the numerical value of $\Lambda^{(10)}$ has been computed in $|2|$ and is prsitive. Com. pactifying to $D$ dimensions on a that toms, one obtains the $D$ dimensional effective 


$$
S_{D}=\int d^{D} x \sqrt{-g^{(D)}}\left(V R^{(D)}-V \Lambda^{(10)}\right)
$$

where $V$ is the volume of the torus. (This effective action is relevant on scales large relative to the string scale $\sqrt{a^{\prime}}$ but small relative to $\phi_{0}^{-1} \sqrt{\alpha^{\prime}}$. At the latter scale, the currature of the background geometry becomes relevant.) $V_{\Lambda}{ }^{(10)}$ increasea as $V \rightarrow \infty$. Howerer it is wrong to conclude from this that the dynamics force us to imaller $V$. In fact the opposite is true. To see this, define a new metric

$$
\dot{g}_{a b}=9_{a b} v \frac{1}{b+3}
$$

$S_{D}$ then takes the canonical form

$$
S_{D}=\int d^{D} \times \sqrt{-\dot{j}^{(D)}}\left(\dot{R}^{(D)}-\frac{(D-1)}{(D-2)} \dot{g}^{a b} \nabla_{a} \ln V \nabla_{b} \ln V-\Lambda V^{-\frac{1}{D-1}}\right)
$$

$\ln V$ is a scalar feld in $D$ dimensions with a canonical kinetic term (up to a constant factor.) Its potential pushes it out to larger $V$ for any finite value of $V$, and $V=+\infty$ is a stable point for $\Lambda>0$. This is in accord with the general behavior described in reference 15

\section{Duality}

We have seen in the last section that the cosmological constant has the symmetry $\Lambda\left(\Gamma_{\text {mod }}\left(S^{T}\right)\right)=\Lambda\left(\Gamma_{\text {sym }}\left(S^{-1}\right)\right)$. Now all the theories we consider can be obtained by $S O(p, q)$ transformations on a given lattice which can be taken as one of the symmetric ones. The above result thus shows that, at least as far as the one loop vacuum energy is concerned, there is a duality between the theories $\Gamma_{\text {sym }}\left(S^{T}\right)$ and $\Gamma_{\text {sym }}\left(S^{-1}\right)$, in the sense that they give the same $A$. (A duality of this type was noticed in the context of bosonic strings in [16].) This result, as we shall show below, generalizes to the full theory.

For the case of $D=9$, the parameter $e^{\beta}$ is proportional to the radius $R$ of the torus involved in the compactification, so the duality gives a mapping between physics at large and small radii. More generally we have a duality between $\Gamma_{\text {sym }}\left(S^{T}\right)$ and $\Gamma_{5 y m}\left(S^{-1}\right)$, where the parameters of $S \in S O(p, q)$ give $a$ measure of the radii of the various tori as well as expectation values for the other fields. This duality generalizes to all the scattering amplitudes and provides a complete mapping between the two theories. (In fact, it applies equally well to the untwisted theorice coneidered by Narain.)

The duslity mapping can be defined in terms of the vertex operators. These are labelled by $\left(k^{\prime}, \dot{k}^{\prime}\right) \in \Gamma$ in addition to the orcillator labels $\{n\}$ and the $D$ dimensional momentum $p$. The vertex $V\left(k^{I}, \tilde{k}^{I},\{n\}, p\right)$ of $\Gamma_{\text {sym }}\left(S^{T}\right)$ is computationally equivalent to $V\left(k^{I},-\tilde{k}^{I},\{n\}, p\right)$ of $\Gamma_{5 y m}\left(S^{-1}\right)$ because the minus sign in front of $\dot{k}^{I}$ is compensated for by a minus sign in the $\dot{X}^{I}$ mode expansion on $S^{T}$ relative to $S^{-1}$. The amplitudes can be calculated in a covariant operator formalism. The invariance of the propagator together with the sbove equivaleace of the vertex operators shows that there is a corresponding mapping of the various scattering amplitudes, i.e., the tree level $S$-matrix elements on $\Gamma_{\text {rym }}\left(S^{T}\right)$ and $\Gamma_{\text {rym }}\left(S^{-1}\right)$ are related by

$$
A\left(k^{I}, \dot{k}^{I},\{n\}, p, S^{T}\right)=A\left(k^{I},-\bar{k}^{I},\{n\}, p, S^{-1}\right)
$$

The mapping relates states of equal mass. The higher loop amplitudes can be con. structed from these by use of unitarity relations and factorization. Thus the duality relation (17) extends to all orders in string perturbation theory

It is instructive to consider this duality in a little more detail for the simplest case, viz. compactification on a circle of radius $R$ to $D=9$. For large values of $R$, we can use the field theory approximation. As $R \rightarrow \infty$, the massive Kaluza-Klein states move down to zero mass and the theory becomes effectively ten dimensiona. How can we get the same spectrum, as implied by duality, for $R \rightarrow 0$ ? In this case, the Kaluza-Klein states get more and more massive. Howerer, the monopole or solitonic states which wrap around the circle move down to zero mass and the theor again

- The vertex upcrators, in addition to polarization tensors and $e^{i k} X$, inrolve the operator cocycles $C(L, L)$. From the definition of $C(L, L)$, one can see that they can be chosen to be invariant, i.e., $C(L,-\dot{L})=C(L, L)$. 
becomes effectirely ten dimensional. These solitonic states are related by the duality transformation to the low mass Kaluza-Klein states at large $R$.

The duality we find is similar to the Krabiers-Wannier duality of two dimensional spin ayatems where there is a mapping between high and low temperatures (rather than large and small radii) [8]. The theory is self dual at the critical point. It is also reminiscent of charged particle-monopole duality where inversions of the type $R \rightarrow \frac{1}{k}$ play a key role. In field theory examples where one has such a duality, e.g. $N=4$ and $N=2$ supersymmetric gauge theories, the mapping also relates strong and weak coupling regimes and the self dual point is again a critical point, i.e. the $\beta$ function is sero [9]. We expect our duality to extend beyond the toroidal compactifications considered here and to be of more general validity in string theories.

This research was supported in part by National Science Foundation grants PHY. 82-1785s and PHY-8313324, and by funds from the National Aeronautics and Space Administration.

\section{Appendix : Modular Invariance}

This appendix provides some mathematical details in the proof of modular invariance of the one loop partition function. As always, let $\Gamma$ be a Lorentzian even self dual lattice and let $\delta^{2}-\dot{\delta}^{2}$ be even. Recall the definitions given in the text:

$$
\begin{aligned}
& A_{-}(r) \equiv \frac{1}{2} z^{-1 / 6} \prod_{n=1}^{\infty}\left(1+z^{n-\frac{1}{2}}\right)^{8}=\frac{1}{2}(2 \pi)^{4 / 3} \frac{\theta_{3}(0, r)^{4}}{\theta_{1}^{\prime}(0, r)^{4 / 3}} \\
& A_{+-}(r) \equiv-8 z^{1 / 3} \prod\left(1+z^{n}\right)^{8}=-\frac{1}{2}(2 \pi)^{4 / 3} \frac{\theta_{2}(0, r)^{4}}{\theta_{1}^{\prime}(0, r)^{4 / 3}} \\
& A-+(r) \equiv-\frac{1}{2} z^{-1 / 6} \prod\left(1-z^{n-\frac{1}{2}}\right)^{8}=-\frac{1}{2}(2 \pi)^{4 / 3} \frac{\theta_{4}(0, r)^{4}}{\theta_{1}^{\prime}(0, r)^{4 / 3}} \\
& B(r) \equiv \frac{1}{z^{1 / 3}} \prod\left(\frac{1}{1-z^{n}}\right)^{24}\left(\frac{1}{1-z^{n}}\right)^{8}=\eta(r)^{-24} \overline{\eta(r)}^{-8} \\
& C_{--}(r) \equiv \sum_{(L, \tilde{L}) \in \Gamma} z^{\frac{1}{2}(L+\delta)^{2}} \bar{z}^{\frac{1}{2}(\bar{L}+\dot{\delta})^{2}}(-1)^{2(L \cdot \delta-\dot{L} \cdot \tilde{\delta})}
\end{aligned}
$$

$$
\begin{aligned}
C_{+-}(r) & \equiv \sum_{\Gamma} z^{\frac{1}{3} L^{2}} z^{\frac{1}{i} \dot{L}^{2}}(-1)^{2(L \cdot \delta-\dot{L} \cdot \dot{\delta})} \\
C_{-+}(r) & \equiv \sum_{\Gamma} z^{\frac{1}{2}(L+\delta)^{\prime}} \sum_{z}^{\frac{1}{3}(\dot{L}+\dot{\delta})^{2}} \\
x & \equiv e^{2 \pi i r}
\end{aligned}
$$

From the properties of $\theta$ functions discussed in reference 17, it easily follow that

$$
\begin{aligned}
A_{-+}(r) & =A_{+-}\left(-\frac{1}{r}\right) \\
A_{--}(r) & =A_{--}\left(-\frac{1}{r}\right) \\
B(r) & =r^{12} B\left(-\frac{1}{r}\right)
\end{aligned}
$$

We can determine the transtormation rules for $C_{-+}$and $C_{+-}$using the identity

$$
\begin{aligned}
\sum_{(L, L)} \exp \left[i \pi r\left(L-\frac{Q}{r}\right)^{2}-i \pi r\left(\bar{L}-\frac{\dot{Q}}{r}\right)^{2}\right] & =(-i r) \frac{-\left(\frac{\pi r-D)}{r}(i r) \frac{-(10-D)}{r}\right.}{} \\
& \sum_{(L, L)} \exp \left[\frac{-i \pi}{r}(L-Q)^{2}+\frac{i \pi}{r}(\dot{L}-\dot{Q})^{2}\right] \exp \left(\frac{i \pi}{r} Q^{2}-\frac{i \pi}{r} \dot{Q}^{2}(A 6)\right.
\end{aligned}
$$

for any vector $Q$. (The derivation for Euclidean self dual lattices in reference 1 is easily extended to the Lorentrian case.) We find

$$
C_{-+}(r)=(-i r)^{-\frac{2 t-0}{r}}(i r)^{-\frac{1 Q-0}{2}} C_{+-}\left(-\frac{1}{r}\right)
$$

The last equation we need is

$$
C_{--(r)}=(-i r)^{-\frac{18-Q}{i}}(i r)^{-\frac{10-Q}{i} Q} C_{--}\left(-\frac{1}{r}\right)
$$

Assuming this for the moment, we prove modular invariance of $A$, as given in equations (8) and (9). The measure $\eta^{-2} d^{2} r$ is invariant under both $r \rightarrow r+1$ and $r \rightarrow-\frac{1}{r}$. From equations (A1) to (A5), the integrand,

$$
r_{2}^{-\frac{Q-1}{2}} B \cdot\left\{\tilde{A}_{--} C_{--}+\tilde{A}_{-+} C_{-+}+\tilde{A}_{+-} C_{+-}\right\}
$$

is invariant under $r \rightarrow-\frac{1}{r}$ since $r_{2} \rightarrow \frac{r_{2}}{|r|^{2}}$. It is also easy to see that $r \rightarrow r+1$ has the sole effect of interchanging the $(--)$ and $(-+)$ terms. 
Finally, we wish to prove equation (A8). For simplicity, take $\Gamma$ to be a Euclidean elf dual lattice in $\mathbf{R}^{N}$. Then (A8) amounts to

$$
\sum_{\Gamma} t^{1}(L+\delta)^{\prime}(-1)^{2 L \cdot \theta}=\left(-i r F^{N / 2} \sum_{\Gamma} x^{\prime} \frac{1}{3}(L+\delta)^{2}(-1)^{2 L \cdot \delta}\right.
$$

where $s^{\prime}=\exp \left(\frac{-2 \pi i}{f}\right)$

We shall need to use the lattice $\Gamma_{\text {even }}=\{L \mid L \cdot \delta \in \mathbf{Z}\}$, where $2 \delta \in \Gamma$ and $\delta^{2} \in \mathbf{Z}$ It dual is amply the lattice generated by $\delta$ and the basis vectors of $\Gamma$, namely,

$$
r_{\text {even }}^{*}=\Gamma \cup\{\Gamma+\delta\}
$$

Consider the following function on $\mathbf{R}^{N}$ :

$$
\begin{aligned}
f(X) & =\sum_{L \in \Gamma} \exp i \operatorname{xr}(L+X)^{2} \cdot \frac{1}{2}[1+\exp i 2 \pi L \cdot \delta] \\
& =\sum_{L \in \Gamma} \exp i \operatorname{ixr}(L+X)^{2}
\end{aligned}
$$

$f$ is periodic on $\Gamma_{\text {eren }}$ so we can take its Fourier transform, defined on $2 \pi \Gamma_{\text {even }}^{\prime \prime}$

$$
f\left(2 \pi L^{*}\right) \equiv \sum_{L \in \Gamma_{\text {man }}} \frac{1}{\operatorname{vol}\left(\Gamma_{\text {even }}\right)} \int_{F_{\text {erea }}} d^{N} X \exp i \pi r(L+X)^{2} \exp i 2 \pi L^{*} \cdot X
$$

$=\frac{1}{\operatorname{rol}\left(\Gamma_{\text {evea }}\right)} \int_{\mathrm{R}} d^{N} X \exp i \pi r X^{2} \cdot \exp i 2 \pi L^{*} \cdot X$

$$
=\frac{1}{2} \cdot(-i r)^{-N / 2} \exp -\frac{i \pi}{r}\left(L^{*}\right)^{2}
$$

where $\operatorname{vol}\left(\Gamma_{\text {mes }}\right)=2$ is the volume of the fundamental cell $F_{\text {even }}$ of $\Gamma_{\text {even }}$. (Recall that $\operatorname{vol}(\mathrm{r})=1$.) Reinverting and using $(\mathrm{A} 10)$, we obtain

$$
\begin{aligned}
f(X) & =\frac{1}{2}(i r)^{-N / 2} \sum_{L \cdot \in \Gamma_{\text {eren }}^{*}} \exp -\frac{i \pi}{r}\left(L^{*}\right)^{2} \cdot \exp -i 2 \pi L^{*} \cdot X \\
& =\frac{1}{2}(-i r)^{-N / 2}\left\{\sum_{L \in \Gamma} \exp -i \pi r L^{2} \cdot \exp (-i 2 \pi L \cdot X)\right. \\
& \left.+\sum_{L \in \Gamma} \exp -\frac{i \pi}{r}(L+\delta)^{2} \cdot \exp -i 2 \pi(L+\delta) \cdot X\right\}
\end{aligned}
$$

We can now extract equation (A9) by setting $X=\delta$ :

$$
\begin{aligned}
f(\delta) & =\frac{1}{2}(-i r)^{-N / 2} \sum_{L \in \Gamma} \exp \left(-\frac{i \pi}{r} L^{2}\right) \exp (-i 2 \pi L \cdot \delta) \\
& +\frac{1}{2}(-i r)^{-N / 2} \sum_{L \in \Gamma} \exp \left(-\frac{i \pi}{r}(L+\delta)^{2}\right) \exp (-i 2 \pi L \cdot \delta) \\
& \equiv \sum_{L \in \mathrm{r}} \exp i \pi r(L+\delta)^{2} \cdot \frac{1}{2}[1+\exp i 2 \pi L \cdot \delta]
\end{aligned}
$$

We already know from (A6) that

$$
\frac{1}{2} \sum_{L \in \Gamma} \exp i \pi r(L+\delta)^{2}=\frac{1}{2}(-i r)^{-N / 2} \sum_{L \in \Gamma} \exp -\frac{i \pi}{r} L^{2} \cdot \exp i 2 \pi L \cdot \delta
$$

Subtracting this from both sides of (A12) yields

$$
\sum_{\Gamma} \exp i \operatorname{rr}(L+\delta)^{2}(-1)^{2 L \cdot \delta}=(-i r)^{-N / 2} \sum_{r} \exp -\frac{i \pi}{r}(L+\delta)^{2}(-1)^{2 L \cdot}
$$

which is precisely (A9). The extension of this argument to the Lorentzian case is straightforward 


\section{References}

$=$

1. L.J. Dixon and J.A. Harvey, String Theories in Ten Dimensione Without Spacetime Supersymmetry, Princeton preprint, February 1986

2. L. Nivarez-Gaume, P. Ginsparg, G. Moore and C. Vafa, An $0(16) \times 0(16)$ Heterotic String, Harvard preprint HUTP-86/A013.

3. N. Seiberg and E. Witten, Spin Structures in String Theories, LAS preprint, Pebruary 1986.

4. K. S. Narain, Phys. Lett. 169B, 4 (1986).

5. B. Witten, New Lssues on Manifolds of SU(3) Holonomy, Princeton preprint, 1985.

6. A.Strominger, Superstrings with Toroiom, Sants Barbara ITP preprint, 1986.

7. K.S. Narain, M. Sarmadi, E. Witten, in preparation.

8. D.J. Grose, J.A. Harvey, E. Martinec and R. Rohm, Phys. Rev. Lett. 54, 502 (1985), Nucl. Phys. B256, 253 (1985), and Nucl. Phys. B267, 75 (1986).

9. J. B. Kogut, Rev. Mod. Phys. 51,659 (1979). R. Savit, Rev. Mod. Phys. 52 , 453 (1980).

10. C. Montonen and D. Olive, Phys. Lett. 72B, 117 (1977). E. Witten and D. Olive, Phys. Lett. 78B, 97 (1978). H. Osborn, Phys. Lett. 83B, 321 (1979).

11. M. Green and J. Schwarz, Phys. Lett. 149B, 117 (1984).

12. R. Rohm, Nucl. Phys. B237, 553 (1984); J. Polchinski, Comm. Math. Phys. 101, 37 (1986).

13. P. Candelas, G. Horowitz, A. Strominger and E. Witten, Nucl. Phys. B285, 48 (1985). C. G. Callan, E. J. Martinec, M. J. Perry and D. Friedan, Nucl.
Phys. B262, 593 (1985). A. Sen, in: Unified String Theories, eds. D. Gross and M. Green (World Scientific 1986); Phys. Rev. D32, 2102 (1985). E. S. Pradkin and A. A. Tseytlin, Phys. Lett. 158B, 316 (1985): Pbys. Lett. 160B, 69 (1985); Nucl. Phys. B261, 1 (1985).

14. W. Fischler and L. Susskind, Dilaton Tadpoles, String Condensates and Seale Invariance, University of Texas preprint UTTG-37.85.

15. M. Dine and N. Seiberg, Phys. Rev. Lett. 55, 366 (1985); Th the Superstring Weakly Coupled?, LAS preprint, July 1985.

16. N. Sakai and I. Senda, Vacuum Energies of String Compactified on Torus. Tokyo Inst. of Tech. preprint TIT/HEP-88, 1985.

17. A. Erdelyi et al., Higher Transcendental Functions, vol. 2 (MeGram Hill, New York, 1958). 